\title{
Pulmonary Function Index Comparisons Depending on Various Postures of Stroke Patients
}

\author{
Kyung-Soo Lee, PT $\cdot$ Myung-Mo Lee, PT, $\mathrm{PhD}^{1 \dagger}$ \\ Department of Physical Therapy, Graduate School of Health and Medicine, Daejeon University \\ ${ }^{1}$ Department of Physical Therapy, Daejeon University
}

Received: November 27, 2018 / Revised: November 30, 2018 / Accepted: December 17, 2018

(c) 2019 J Korean Soc Phys Med

\begin{abstract}
| Abstract |
PURPOSE: To prevent secondary complications from decreased pulmonary functions and promote neurological recovery, identification of respiratory capacity change patterns depending on different postures of stroke patients and investigation of their properties are needed for active rehabilitation. Therefore, this study was conducted to investigate the changes in vital capacity in response to different positions and to implement the results as clinical
\end{abstract} data.

METHODS: A respiratory function test was administered to 52 patients with stroke in the sitting, supine, paretic side lying, and non-paretic side lying positions. Pulmonary function indexes used for comparison were forced vital capacity (FVC), forced expiratory volume at 1 second (FEV1), forced expiratory flow 25-75\% (FEF 25-75\%), and maximum voluntary ventilation (MVV). One-way repeated

†Corresponding Author: Myung-Mo Lee mmlee@dju.kr, https://orcid.org/0000-0002-2192-1701

This is an Open Access article distributed under the terms of the Creative Commons Attribution Non-Commercial License (http://creativecommons.org/licenses/by-nc/3.0) which permits unrestricted non-commercial use, distribution, and reproduction in any medium, provided the original work is properly cited.
ANOVA was used for analysis, and post hoc analysis was conducted using least significant difference (LSD).

RESULTS: All pulmonary function indexes were measured in the order of sitting, paretic side lying, supine, and non-paretic side lying positions. Excluding the FEF25-75\% and MVV of the supine compared with the paretic side lying position, all other pulmonary function indexes differed significantly $(\mathrm{p}<.05)$.

CONCLUSION: There are differences in pulmonary function indexes depending on different postures of stroke patients, and the study showed that the non-paretic side lying position yielded the greatest effect on lung ventilation mechanisms. Based on these results, appropriate postures need to be considered during physical therapy interventions for stroke patients.

Key Words: Respiration, Respiratory function tests, Spirometry, Stroke, Ventilation

\section{Introduction}

Stroke is a neurological symptom that is induced by the interruption of blood supply because of the blockage or rupture of brain blood vessels[1]. Stroke affects 1.5 million people every year, a third of whom are left with 
a lasting disability[2]. Hemiplegia resulting from stroke leads to overall muscle contraction and weakness on the hemi side, and $70 \%$ of the patients are left with chronic disability[3]. In addition, voluntary motor function and coordination functions of trunk muscles are injured, resulting in abnormality in posture, muscle tone, and motor control[4]. Decreased motor control ability is a major factor that decreases general performance and activities during daily living[5]. Additionally, leaned nonuse after stroke tends to lead to an inactive lifestyle, which gradually decreases everyday physical activities and the range of social participation, increasing deterioration of physical functions including gait and balance[6].

Long-term bedside resting and decreased physical activities may induce secondary complications such as pressure sores, osteoporosis, circulation dysfunction of the lower limbs, nutrition and metabolism dysfunction, endocrine dysfunction, mental issues, and neurological complications[7]. In addition, physical weakening such as nonuse atrophy and contraction affects respiratory muscles, lung tissues, and cardiovascular structures[8]. These changes in the interrelationships of the structures result in gradual decreases of cardiopulmonary functions and gas exchange, which leads to decreased vital capacity, lung volume, trachea obstruction, fluid velocity, and oxygen saturation in the arteries[9-11]. Weakening and paralysis of the diaphragm and respiratory muscles leads to contraction of the thoracic cage and decreased lung elasticity[12,13], and these decreases in cardiopulmonary functions have decreasing effects on total respiration volume and capacity, including forced vital capacity (FVC) and forced expiratory volume in one second (FEV1) [14]. This results in decreased sputum excretion, which leads to complications such as pneumonia and atelectasis. Therefore, appropriate postural changes are suggested in clinical settings to distribute pressurized areas of the body, as well as increase oxygen supply and delivery to minimize aspiration risks and make expulsion of lung discharges easier for compensation of insufficient lung capacity[15]. Decreased cardiopulmonary functions make a person feel easily fatigued during physical activities, which restricts independent performance of activities of daily living[16]. Early active participation in rehabilitation is recommended for stroke patients, but rehabilitation therapies for functional recoveries may induce physical fatigue due to decreased vital capacity[13,17,18].

Recent related studies report contraction of the thoracic cage on the affected side and weakness of respiratory muscles in acute stroke patients with the tendency for restrictive lung diseases or hemiplegic patients with chronic stroke[12,13,19,20]. Specific patterns of most post-stroke pulmonary function decreases are explained by direct causes such as poor diaphragm motor functions and respiratory muscle weakness.

Shin et al.[21] suggested that changes in the autonomous nervous system in response to alterations in postures during physical therapy implementation be considered in patients with neurological conditions. Additionally, studies have investigated the breathing capacity depending on postures with normal adults and the elderly[8,22], and compared breathing capacity changes depending on postures of cardiovascular and neurological patients such as chronic pulmonary diseases and spinal cord injury[23,24]. However, despite asymmetrical weakness of the diaphragm and accessory respiratory muscles because of hemiplegia in stroke patients, there are insufficient studies comparing vital capacity changes that consider the affected side in stroke patients who require training in different postures[13]. To prevent secondary complications from decreased pulmonary functions and promote continuous neurological recovery, identification of vital capacity change patterns depending on different postures of stroke patients and investigation of the properties are needed for active rehabilitation therapies. In this study, we will investigate the changes in the vital capacity depending on different postures of stroke patients and suggest appropriate postures 

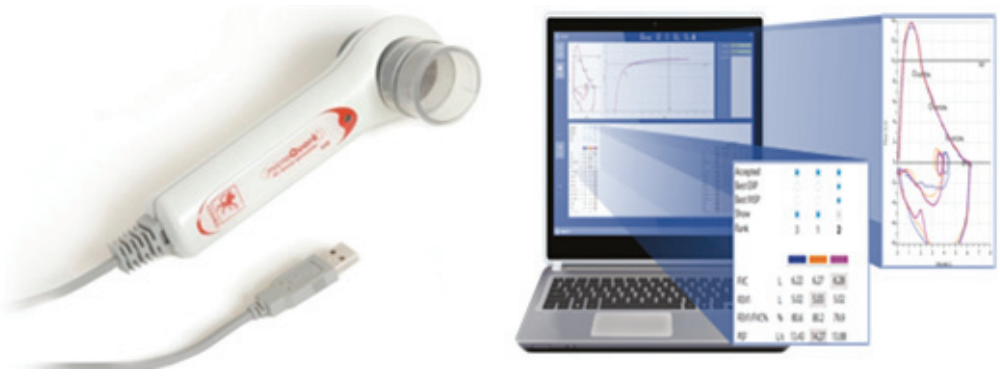

Fig. 1. Device used to measure pulmonary functions and analysis software

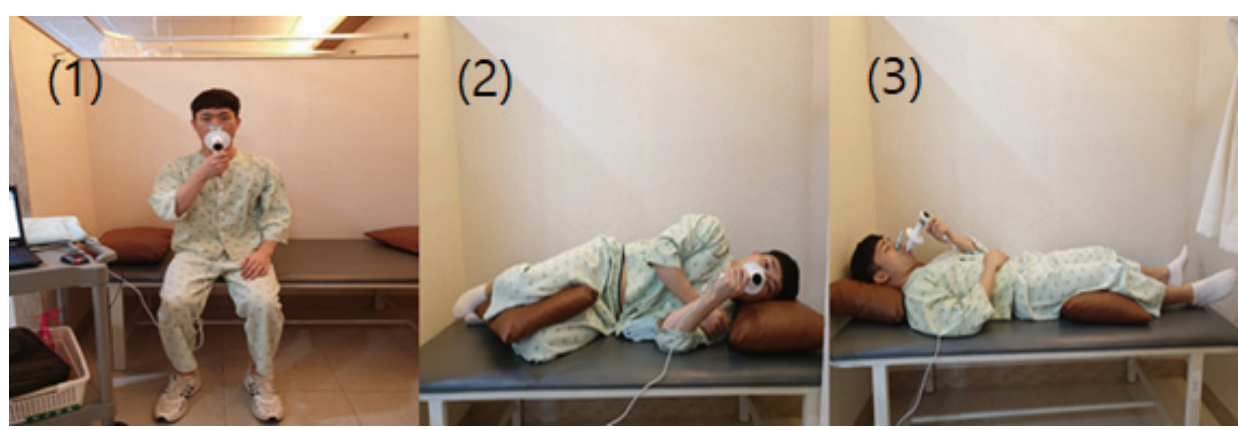

Fig. 2. Measurement of pulmonary function test in different positions

(1) sitting position, (2) paretic or non-paretic side lying position, (3) supine position

that should be considered when applying high intensity repetitive strength exercise or rehabilitation for the functional enhancement. Overall, this study investigates the changes in vital capacity with variations in posture of patients with hemiplegia due to stroke and utilizes the outcomes as clinical data.

\section{Research Method}

\section{Participants}

A total of 70 patients who were diagnosed with stroke more than 6 months ago and who are in the chronic stage of hemiplegia were recruited from $\mathrm{H}$ hospital in D city. The inclusion criteria were patients who are able to change postures independently, maintain at least 15 minutes sitting, lying on both the affected and non-affected side, and in the supine positions, and have scored higher than 24 in
MMSE-K. Patients with a history of tuberculosis, pulmonary conditions such as asthma, orthopedic conditions such as congenital thoracic cage deformity or rib fracture, history of surgery requiring abdominal incision, and uncontrolled cardiovascular diseases were excluded from the study. All of the participants were informed of the study purpose and procedures, and only those who agreed to voluntary participation and signed a written consent form were recruited. This study was approved by the ethical committee of D University (1040647-201804- HR-004-03).

\section{Procedures}

This investigation was a cross-sectional study conducted from May to August in 2018. The G-power 3.13 software was used to calculate the sample size. The power and alpha level were set as 0.08 and 0.5 , respectively, while the effect size was set at 0.4 according to a preliminary study that 
compared the FVC between the sitting and supine positions. Eighteen patients from the initial 70 participants were excluded because they did not meet the inclusion criteria, resulting in 52 participants being included. The participants were administered vital capacity tests in the sitting and supine positions, as well as while lying sideways on both the affected and non-affected side to investigate changes in vital capacity with variations in body position. Prior to the measurement, the participants were given an explanation of the process and precautions associated with the vital capacity test. To eliminate the effects of practice, measurements were first taken in the sitting position, then at randomly selected positions. Vital capacity measurement was conducted between $6 \mathrm{PM}$ and $8 \mathrm{PM}$ after all routines were over. One participant was analyzed for vital capacity in all of the measurement positions each day.

\section{Measurement tools}

A spirometer (microQuark, Cosmed, Italy) was used to measure vital capacity, and Omnia software (Omnia software, v1.6.4, Cosmed, Italy) was used to collect the data (Fig. 1). To make the measurement of vital capacity more accurate, room temperature was set to $20^{\circ} \mathrm{C}$ with a humidity of $50 \%$. A nose clip was used to prevent air inspiration and expiration through the nose during the measurement process, and participants were educated on being cautious not to let the air slip out through the mouth piece. If accurate data was not recorded because of air ventilation through the nose or the mouth piece, another attempt at measurement was made.

When measuring in the sitting position, the hip and knee joint were maintained in 90 degrees and the chair height was adjusted so that both soles of the feet touched the floor. To minimize any compensations of the trunk during inhalation and exhalation, the participants were asked to maintain an upright posture. In both side lying positions, vital capacity was measured after placing appropriate pillows between the knees, under the lumbar spine, and by the head to minimize any shape deformities from the body weight of the thoracic cage and to maintain levelness of the whole spine. Vital capacity measurement in the supine position was made after placing pillows under the knees and head to maintain a comfortable posture (Fig. 2).

The vital capacity test measured the forced vital capacity (FVC), forced expiratory volume at 1 second (FEV1), forced expiratory flow 25-75\% (FEF 25-75\%), and maximum voluntary ventilation (MVV). FVC is the vital capacity measurement variable when air is inhaled as deeply as possible and exhaled as much as possible. FEV1 is the maximum exhalation volume for 1 second after the maximal inspiratory level. The FEF $25-75 \%$ describes the middle $50 \%$, excluding the initial and terminal $25 \%$ of the total vital capacity. MVV is the maximum voluntary ventilation for 1 minute, where the ventilation is measured for 12 seconds and the variable is multiplied with 5. Measurements were made three times in the same position and the greatest measurement value was taken into consideration. Five minutes of rest was given in between each trial[25]. In cases in which participants reported dizziness, vomiting, headache, or breathing difficulties, the trial was stopped immediately and continued after sufficient resting time only if the participant agreed to continue. Vital capacity tests were conducted by one physiotherapist with experience in vital capacity tests, and data collection and recording were done by an assisting researcher.

\section{Analysis}

This study used SPSS win 19.0 to analyze the data. General characteristics of the participants were analyzed using mean and standard deviation values through descriptive statistics, and the Shapiro-Wilk test was used to confirm the normal distribution. Each variable measured was used to calculate the mean and standard deviation, and one-way repeated ANOVA was used to identify differences in the measurement values of respiratory functions at different positions. Post-hoc comparisons were 
Table 1. Comparison of pulmonary function test results when in different positions

\begin{tabular}{cccccc}
\hline & Sitting & Supine & $\begin{array}{c}\text { Paretic } \\
\text { Side lying }\end{array}$ & $\begin{array}{c}\text { Non-paretic } \\
\text { Side lying }\end{array}$ & F/p \\
\hline FVC (1) & $2.59 \pm 0.73^{\mathrm{a}}$ & $2.34 \pm 0.76^{\dagger}$ & $2.46 \pm 0.73^{\dagger \ddagger}$ & $2.29 \pm 0.67^{\dagger \S \S}$ & $18.324 / .000$ \\
FEV1 (l) & $2.19 \pm 0.64$ & $1.97 \pm 0.63^{\dagger}$ & $2.04 \pm 0.63^{\dagger \ddagger}$ & $1.89 \pm 0.60^{\dagger \ddagger}$ & $14.666 / .000$ \\
FEF25-75\% (1/S) & $2.52 \pm 0.95$ & $2.19 \pm 0.79^{\dagger}$ & $2.24 \pm 0.84^{\dagger}$ & $2.07 \pm 0.85^{\dagger \S}$ & $9.071 / .000$ \\
MVV (l) & $47.98 \pm 17.19$ & $41.48 \pm 16.94^{\dagger}$ & $42.99 \pm 17.75^{\dagger}$ & $38.96 \pm 16.00^{\dagger \S \S}$ & $20.165 / .000$ \\
\hline
\end{tabular}

${ }^{\mathrm{a}}$ Mean \pm standard deviation, FVC; forced vital capacity, FEV1; forced expiratory volume in 1 second, FEF; forced expiratory flow, MVV; maximum voluntary ventilation

${ }^{\dagger}$ significant difference compared to sitting $(\mathrm{p}<.05)$

${ }^{\ddagger}$ significant difference compared to supine $(\mathrm{p}<.05)$

$\S_{\text {significant difference compared to paretic side lying }(\mathrm{p}<.05)}$

conducted using the Fisher's least significant difference (LSD) test. Statistical significance was set to $\mathrm{p}<.05$.

\section{Results}

Pulmonary function of 52 stroke patients (male $=25$, female $=27$ ) was measured. The mean age of the participants was $57.89 \pm 12.69$ years, the mean height was $163.68 \pm 8.98$ $\mathrm{cm}$, and the mean weight was $65.50 \pm 9.47 \mathrm{~kg}$. Twenty-two participants were diagnosed with cerebral hemorrhage, and 30 were diagnosed with cerebral infarction. Additionally, 24 participants had left hemiplegia and 28 had right hemiplegia. The mean onset of conditions in the participants was $31.04 \pm 23.08$ months.

The results of the pulmonary function test showed that FVC, FEV1, FEF25-75\%, and MVV were all high when participants were in the sitting, paretic side lying, supine, and non-paretic side lying positions, respectively. The pulmonary function indexes in different postures revealed statistically significant differences $(\mathrm{p}<.05)$. Post hoc analysis showed that all indexes (FVC, FEV1, FEF25-75\%, MVV) measured in the sitting position differed significantly from those measured in the supine, paretic side lying, and non-paretic side lying positions $(\mathrm{p}<.05)$. FVC and FEV1 measured in the supine position differed significantly from those measured when lying on the paretic side $(\mathrm{p}<.05)$, and all indexes (FVC, FEV1, FEF25-75\%, MVV) differed significantly between the supine and non-paretic side lying positions $(\mathrm{p}<.05)$. All pulmonary function indexes measured in the paretic side lying position differed significantly $(\mathrm{p}<.05)$ from those measured when lying on the non-paretic side (Table 1).

\section{Discussion}

This study investigated differences in pulmonary function of stroke patients depending on posture. The pulmonary function indexes occurred in decreasing order of sitting, paretic side lying, supine, and non-paretic side lying positions, and showed statistically significant differences by the positions excluding FEF25-75\% and MVV of supine compared with paretic side lying positions. The human body is affected by various mechanisms that maintain homeostasis of respiratory functions even when posture changes. Medullary reticular formation and the vestibular system control respiratory muscle activities appropriately depending on different postural changes and movements of the body[26]. However, gravitational factors (e.g., ventilation and perfusion, shifting of abdominal organs, same-side contraction of the diaphragm) following 
postural changes and non-gravitational factors (restricted mobility of the thoracic wall, respiratory muscle weakening) may lead to difference in lung ventilation[27]. These factors, when accompanied by respiratory diseases such as weakening of respiratory muscles and lung parenchyma injury, may result in decreased predicted ventilation and yield restrictive ventilatory defects. Such restrictive ventilation is a common issue in patients with neurological injuries or respiratory muscle weakness, and is therefore a physiological index that needs to be considered when evaluating respiratory complications and during rehabilitation training in various positions.

The vital capacity measured by different postures in normal adults is usually greater when standing than sitting. In a sitting position, the hip joints are flexed to 90 degrees and the lengths of the abdominal muscles are shorter than when standing[28]. Evaluation of the relationship between the muscle length and tensile force has shown that abdominal muscles are in the optimal position when standing, resulting in a beneficial generation of abdominal pressure[29]. According to Cotes et al.[30], vital capacity is $7 \%$ greater when standing than sitting.

However, vital capacity when sitting is greater than when leaning 45 degrees seated or in the supine position. Although seated leaning at 45 degrees is a position on which abdominal muscles are relaxed and able to promote sufficient inhalation[25,31], expansion of the thoracic cage on the dorsal side is restricted against the back of the chair, making the vital capacity lower than when seated upright. In addition, thoracic cage expansion on the dorsal side is even more restricted in the supine position than when seated and leaning 45 degrees because of the gravitational force working against it. Therefore, vital capacity is decreased in the supine position because the volume of blood going through the lungs increases and the gas volume inhaled into the thoracic cage decreases by pressurizing the diaphragm[22,32,33]. These factors are similarly applicable to normal adults and stroke patients. Seo et al.
[34] compared the vital capacities of 20 stroke patients (10 males, 10 females) when sitting, leaning 45 degrees, and in the supine position and found that it was $42 \%$ lower in the supine position than the sitting position. The results of this study demonstrated that all pulmonary function indexes of stroke patients decreased significantly by $10-$ $15 \%$ when in the supine position relative to the sitting position. This discrepancy between studies may be a result of differences in pulmonary function indexes of the participants and in the sample size.

Studies that compared vital capacities in the supine and side lying positions of normal adults have had differing results. Seo and $\mathrm{Cho[22]} \mathrm{investigated} 20$ healthy adults and found that the supine position resulted in significantly greater than lying on the right side. However, Ganapathi and Vinoth[28] reported lying on the side led to greater vital capacity values than being in the supine in 20 healthy males. Similarly, Jenkins et al.[35] reported greater vital capacity values when lying on the side than the supine position. When lying on the side, less gravitational force is applied than when in the supine position, making anterior expansion of the abdomen easier. Additionally, restriction of dorsal thoracic expansion is greater in the supine position than expansion restriction of the thoracic cage on the bottom side in the side lying position, which makes the measured vital capacity greater when lying on the side.

In prior studies comparing the vital capacity of healthy adults while lying on the right and left side, many researchers reported greater vital capacity on the right side $[8,22,28]$, but these differences were not statistically significant. However, Manning et al.[8] hypothesized that there may be vital capacity differences in the left and right lungs depending on the age and the conditions of the heart and lungs when sitting, while Zack et al.[23]. reported that arterial oxygen tension was greater when lying on the left side than on the right in a study of 13 patients with respiratory diseases. However, there was no difference in six healthy adults. The left lung is typically smaller than 
the right lung, and when lying on the left side, there is greater pressure from the heart when the diaphragm contracts. As a result, the vital capacity is greater when lying on the right side. Changes in the size and shape of the lungs occur with age and there may be differences in vital capacity with pressure from the heart. However, that ratio is irregular and there is a lack of evidence to support this[8].

Decrease in lung function of stroke patients initially results from paralysis or weakening of the diaphragm and accessory respiratory muscles. In the chronic stage, activity level is decreased compared to a person without the condition, despite recovery, and continuous weakening of the muscles, contraction of the thoracic cage, and decrease of lung elasticity restricts full expansion of the lungs, resulting in restrictive ventilatory dysfunction. This restrictive ventilatory dysfunction is more obvious in postural changes of stroke patients. The results of this study showed the measured values high in the comparison between supine and paretic side lying positions (excluding FEF25-75\% and MVV), but the measured values were low with statistically significant differences in comparison between supine and non-paretic side lying positions. In addition, lying on the non-paretic side led to significant decreases in all pulmonary function indexes compared to lying on the paretic side. Based on these results, lying on either the paretic or non-paretic side was found to affect abdominal and thoracic expansions in stroke patients. When lying on the paretic side, thoracic expansion on the paretic side is restricted by body weight, but the abdominal and thoracic expansions on the non-paretic side occurs in a relatively normal manner. These findings indicate that restriction of non-paretic thoracic expansion and weakened expansion mechanisms when lying on the non-paretic side allowed the vital capacity to be greater. Additionally, pulmonary function indexes were found to be lower in the supine position because of the weakened ventilation mechanism, which contradicts high measurement of FVC and FEV1 when lying on the side compared to the supine position. When lying on the side on the paretic side, the reason for the lack of differences in the FEF25-75\% and MVV between individuals lying on their sides and those in the supine position may be because, although randomly assigned, many participants were evaluated while lying on the paretic side last and fatigue levels may have increased from vital capacity measurements.

Previous studies have investigated factors responsible for the functional decrease of lung capacity in stroke patients, but there is a lack of evidence regarding changes in vital capacity depending on different postures. Specifically, this is the first study that compared vital capacities in paretic and non-paretic side lying positions. Based on the results presented herein, it is necessary to suggest a proper posture when considering the vital capacity during rehabilitation treatment of stroke patients. However, it should be noted that this study had several limitations. Specifically, not all participants had restrictive ventilation, which makes generalizations difficult. Some participants also complained of changes in muscle tone because of postural changes, inadequate ventilation switching, and inadvertent forced expiratory restriction. Discussions of vital capacity changes from weakened respiratory muscles or restricted thoracic cage expansion are difficult, since the participants were in the chronic stage of stroke and had an onset greater than 6 months. Results of strength measurement of respiratory muscles that relate to inhalation and exhalation and additional neurophysiological discussions are needed. Additionally, future studies that investigate changes in the vital capacity of stroke patients in the acute and subacute stages are needed for comparison.

\section{Conclusion}

This study compared the FVC, FEV1, FEF25-75\%, and MVV values of chronic stroke patients with an onset of over 6 months for vital capacity depending on different 
postures. The values were higher in patients while sitting, lying on the paretic side, in the supine position and while lying on the non-paretic side, and lying on the non-paretic side had the greatest effect on lung ventilation mechanisms the Based on these results, it is suggested that appropriate postures for ventilation abilities be considered when administering physical therapy interventions to stroke patients.

\section{References}

[1] Harvey R, Roth E, Yu D. Rehabilitation in stroke syndromes. PM\&R. Philadelphia: Saunders Elsevier. 2007:1175-12.

[2] Geyh S, Cieza A, Schouten J, et al. ICF Core Sets for stroke. J Rehabil Med. 2004(44 Suppl):135-41.

[3] Duncan PW, Horner RD, Reker DM, et al. Adherence to postacute rehabilitation guidelines is associated with functional recovery in stroke. Stroke. 2002;33(1):167-77.

[4] de Almeida IC, Clementino AC, Rocha EH, et al. Effects of hemiplegy on pulmonary function and diaphragmatic dome displacement. Respir Physiol Neurobiol. 2011; 178(2):196-201.

[5] Andrews AW, Bohannon RW. Distribution of muscle strength impairments following stroke. Clin Rehabil. 2000;14(1):79-87.

[6] Richards CL, Malouin F, Wood-Dauphinee S, et al. Task-specific physical therapy for optimization of gait recovery in acute stroke patients. Arch Phys Med Rehabil. 1993;74(6):612-20.

[7] Grotta JC, Lo EH. Stroke: pathophysiology, diagnosis, and management. Elsevier Health Sciences. 2015.

[8] Manning F, Dean E, Ross J, et al. Effects of side lying on lung function in older individuals. Phys Ther. 1999;79(5):456-66.

[9] Georges R, Saumon G, Loiseau A. The relationship of age to pulmonary membrane conductance and capillary blood volume. Am Rev Respir Dis. 1978;117(6):1069-78.
[10] Zadai CC. Pulmonary physiology of aging: the role of rehabilitation. Top Geriatr Rehabil. 1985;1(1):49-57.

[11] Miner B, Gill TM, Vaz Fragoso CA. Physiology of the Aging Lung. The Aging Lungs: Mechanisms and Clinical Sequelae. World Scientific. 2016.

[12] Lanini B, Gigliotti F, Claudia C, et al. Dissociation between respiratory effort and dyspnoea in a subset of patients with stroke. Clin Sci. 2002;103(5):467-73.

[13] Kim BR, Chun MH, Kang SH. Change of Respiratory Function following Rehabilitation in Acute Hemiplegic Stroke Patients. J Korean Acad Rehabil Med. 2009; 33(1):21-8

[14] Howard R, Rudd A, Wolfe C, et al. Pathophysiological and clinical aspects of breathing after stroke. Postgrad Med J. 2001;77(913):700-2.

[15] Dean E. Effect of body position on pulmonary function. Phys Ther. 1985;65(5):613-8.

[16] Estenne M, Gevenois PA, Kinnear W, et al. Lung volume restriction in patients with chronic respiratory muscle weakness: the role of microatelectasis. Thorax. 1993; 48(7):698-701.

[17] MacKay-Lyons MJ, Makrides L. Longitudinal changes in exercise capacity after stroke. Arch Phys Med Rehabil. 2004;85(10):1608-12.

[18] Ito H, Kano O, Ikeda K. Different variables between patients with left and right hemispheric ischemic stroke. J Stroke Cerebrovasc Dis. 2008;17(1):35-8.

[19] Lanini B, Bianchi R, Romagnoli I, et al. Chest wall kinematics in patients with hemiplegia. Am J Respir Crit Care Med. 2003;168(1):109-13.

[20] Harraf F, Ward K, Man W, et al. Transcranial magnetic stimulation study of expiratory muscle weakness in acute ischemic stroke. Neurology. 2008;71(24):2000-7.

[21] Shin YA, Kim MJ, Ahn JH. The comparison of heart rate variability and blood pressure according to posture and exercise type. Exercise science. 2011;20(3):249-60.

[22] Seo KC, Cho MS. An analysis of pulmonary function in different lying positions in the 20's normal adults. 
J Phys Ther Sci. 2016;28(11):3063-5.

[23] Zack MB, Pontoppidan H, Kazemi H. The effect of lateral positions on gas exchange in pulmonary disease: a prospective evaluation. Am Rev Respir Dis. 1974;110(1): 49-55.

[24] Chen C, Lien IN, Wu M. Respiratory function in patients with spinal cord injuries: effects of posture. Spinal Cord. 1990;28(2):81.

[25] Pryor JA, Prasad AS. Physiotherapy for respiratory and cardiac problems: adults and paediatrics. Elsevier Health Sciences. 2008

[26] Mori R, Bergsman A, Holmes M, et al. Role of the medial medullary reticular formation in relaying vestibular signals to the diaphragm and abdominal muscles. Brain research. 2001;902(1):82-91.

[27] Galvin I, Drummond G, Nirmalan M. Distribution of blood flow and ventilation in the lung: gravity is not the only factor. Br J Anaesth. 2007;98(4):420-8.

[28] Ganapathi LV, Vinoth S. The estimation of pulmonary functions in various body postures in normal subjects. Int J Advances Med. 2015;2(3):250-4.
[29] MoCool F, Leith D. Pathophysiology of cough. Clin Chest Med. 1987;8(2):189-95.

[30] Cotes JE, Chinn DJ, Miller MR. Lung function: physiology, measurement and application in medicine. John Wiley \& Sons. 2009.

[31] Kisner C, Colby LA, Borstad J. Therapeutic exercise: foundations and techniques. Fa Davis. 2017.

[32] Kera T, Maruyama $\mathrm{H}$. The effect of posture on respiratory activity of the abdominal muscles. J Physiol Anthropol Appl Human Sci. 2005;24(4):259-65.

[33] Kwon SE, Lee DY, Hong JH, et al. Effects of various angle on respiration function in adults. Indian J Sci Technol. 2015;8(26).

[34] Seo KC, Lee SE, Lee JH, et al. Comparison of Posture on Respiratory Function in the Stroke Patients according to Changes of Position. J Korean Soc Phys Med. 2011;6(4):381-9.

[35] Jenkins SC, Soutar SA, Moxham J. The effects of posture on lung volumes in normal subjects and in patients pre-and post-coronary artery surgery. Physiotherapy. 1988;74(10): 492-6. 
